\title{
Development of Silicon-based Simulator for Thyroid Surgical Practice
}

\author{
Ren Ota, ${ }^{1 *}$ Ikuo Yamamoto, ${ }^{1,3}$ Tomohiro Obata, ${ }^{1,2}$ Masayuki Baba, ${ }^{1,2}$ Naoto Matsuo, ${ }^{1,2}$ \\ Fumitake Uchida, ${ }^{1,2}$ Murray Lawn, ${ }^{1,3}$ Keitaro Matsumoto, ${ }^{1,2}$ and Takeshi Nagayasu ${ }^{1,2}$ \\ ${ }^{1}$ Medical-Engineering, Hybrid Professional Development Center, \\ Nagasaki University Graduate School of Biomedical Sciences, Nagasaki, Japan \\ ${ }^{2}$ Department of Surgical Oncology, Nagasaki University Graduate School of Biomedical Sciences, Nagasaki, Japan \\ ${ }^{3}$ Division of Mechanical Science, Nagasaki University Graduate School, Nagasaki, Japan
}

(Received June 15, 2019; accepted December 2, 2019)

Keywords: thyroid, echo, 3D printer, silicon

Fine needle aspiration cytology (FNAC) is commonly used and is essential for diagnosing thyroid diseases. Despite its widespread use, it presents a significant barrier for doctors who lack experience with this procedure, owing to the inherent difficulty involved in identifying and avoiding venous arteries such as the internal jugular vein, common carotid artery, and trachea. At this stage, a universal neck simulator supporting FNAC practice has yet to be developed. This project is based on an earlier simulator developed by the authors. However, a number of significant improvements have been made to bring this closer to what could be potentially a practical simulator for thyroid FNAC practice. Improvements have been made in terms of the accuracy of the organs and the addition of muscle tissue, but the most significant issue is the use of a material that does not require special storage, namely, silicone mixed with a number of compounds as required to sonographically and haptically simulate the neck and thyroid.

\section{Introduction}

In regard to thyroid-related medical procedures, it is important to interact with a lifesized model to appreciate the intricacies of this complex structure consisting of the trachea, blood vessels, muscles, and cartilage. Ultrasound and puncture diagnosis, particularly in the examination of thyroid tumors, require skill and experience. Therefore, we are developing a thyroid fine needle aspiration cytology (FNAC) procedure training simulator at our university. ${ }^{(1)}$ On the basis of computer tomography (CT) image scan data of the neck region, various organs have been recreated using 3D printer technology to mimic the neck region, thus allowing doctors who are unexperienced in this procedure to gain experience and increase their skill level.

This practice simulator is combined with the soft molding technology developed by MICOTO Technology. Our goal is to finish the simulator with materials having haptic and sonographic properties that are closer to those of real organs. This allows doctors to obtain experience that is similar to that of examining an actual subject. In regard to the density (hardness) *Corresponding author: e-mail: hare.mabuta25@gmail.com https://doi.org/10.18494/SAM.2019.2459 
of various organs, it is very important to not only provide accurate haptic feedback for the FNAC procedure but also use materials that provide sonographically accurate images. The newly developed silicon-based neck-thyroid FNAC procedure simulator is shown in Fig. 1.

\section{Material and Methods}

The purpose of this research is to prototype a more practical neck phantom using silicon as the base material and various compounds to adjust the sonographic imaging. Regarding the ultrasonic measurements carried out in this research, assuming that the sound velocity of water is about $1483 \mathrm{~m} / \mathrm{s}\left(20^{\circ} \mathrm{C}\right)$ and the sound velocity during echo measurement is about $1560 \mathrm{~m} / \mathrm{s}$, the cross-section aspect ratio is $1: 1$. This is calibrated by the device manufacturer considering that about $70 \%$ of the human body is composed of water. However, the choice of a silicon-based model and the resulting variation in sound velocity cause variations in this aspect ratio. The grayscale characteristics of the echo image reflecting the density of respective organs are controlled by adding various compounds to silicon. The following experiment was conducted to select the most appropriate compounds to accurately reflect the desired grayscale densities.

\subsection{Base material}

The base material was selected on the basis of the following experiment. A $2 \mathrm{~cm}^{3}$ mold was 3D-printed. Three types of silicon with different hardnesses were cast using this mold. Then, a test piece of silicon fixed in a water tank was measured using a linear probe with frequencies of 7.5 to $10 \mathrm{MHz}$. To prevent echo back from the bottom of the tank, the test piece was set at a distance of $X$ from the probe where the distance from the test piece to the bottom varied between $4 \times X$ to $6 \times X$.

\subsection{Grayscale}

Grayscale experiments were carried out by changing the ratio of the following compounds, which were previously investigated at the authors' university. Various ratios of sodium alginate, calcium phosphate, and gluconolactone compounds were mixed with silicon and the resulting sonographic grayscales were measured.

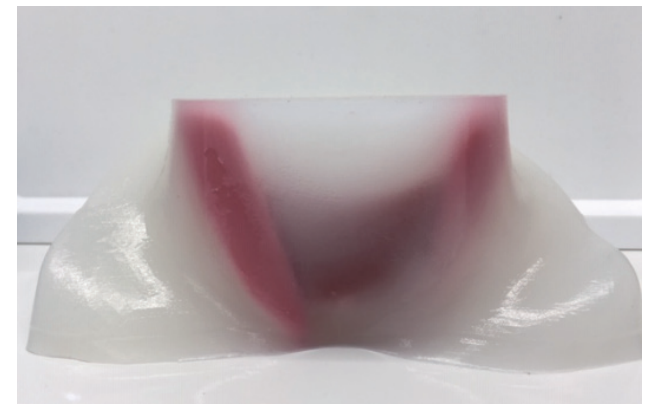

Fig. 1. (Color online) Silicon-based neck model simulator. 


\subsection{Neck model}

By using silicon as the base material mixed with the compounds necessary to provide accurate grayscale imaging, the neck model was created. 3D data were extracted from CT scan images to provide high-resolution 3D data on which to base the model. Each component/organ was processed to produce a 3D-printed mold. Each component/organ was cast with silicon and various ratios of compounds as shown in Table 1 . The echogenicity of blood vessels and tumors is low, blood vessels are thin, and tumors are small, so increased hardness was required (silicon C). The echogenicity of the thyroid is relatively high, and it is necessary to maintain the shape of the thyroid in order to puncture it. Therefore, low-hardness silicon A was used with a 3\% mixture of sodium phosphate. The echo brightness of subcutaneous tissue was slightly high, and it was necessary to maintain the strength and elasticity of subcutaneous tissue. Therefore, low-hardness silicon A was used and calcium phosphate was mixed at $1.25 \%$.

\section{Results}

\subsection{Base material}

Various silicone hardnesses were examined and the resultant aspect ratio errors are shown in Table 2. Silicon A closest to $2 \mathrm{~cm}$ was selected as the base material. An error of $\pm 3.5 \%$ with a maximum error of $0.07 \mathrm{~mm}$ was considered to be acceptable. As increased rigidity is required to reproduce blood vessels and tumors, silicon $\mathrm{C}$ was used as a base material and the model was scaled to $86 \%$ to compensate for the aspect error.

\subsection{Grayscale}

When the proportion of a compound mixed is $1.0 \%$ or less with respect to the amount of silicon, it was not possible to clearly detect the density. Furthermore, when the mixture exceeded $10 \%$, the compound tended to saturate and form a sediment. On the basis of these observations, compound mixtures with proportions between 1.5 and $9.5 \%$ were considered to

Table 1

Simulated organ components.

\begin{tabular}{lccc}
\hline & Ingredient & Particulate & $\begin{array}{c}\text { Percentage } \\
(\%)\end{array}$ \\
\hline $\begin{array}{l}\text { Subcutaneous } \\
\text { tissue }\end{array}$ & Silicon A & Calcium phosphate & 1.25 \\
$\begin{array}{l}\text { Muscle } \\
\text { Thyroid }\end{array}$ & Silicon A & Glucono- $\delta$-lactone & 5 \\
$\begin{array}{l}\text { Anterior } \\
\text { cervical muscle }\end{array}$ & Silicon A & Sodium phosphate & 3 \\
$\begin{array}{l}\text { Blood vessels } \\
\text { Tumor }\end{array}$ & Silicon A & Glucono- $\delta$-lactone & 3 \\
Trachea & Silicon C & - & - \\
Spine & PLA & - & - \\
\hline
\end{tabular}

Table 2

Silicon hardness and maximum error from actual size

\begin{tabular}{lcc}
\hline & Hardness & $\begin{array}{c}\text { Maximum error from } \\
\text { actual size }(\mathrm{mm})\end{array}$ \\
\hline Silicon A & $00-30$ & 0.07 \\
Silicon B & 10 & 0.25 \\
Silicon C & 30 & 0.31 \\
\hline
\end{tabular}


be effective for this development. Regarding the use of liquid colorant, the addition of up to $2 \%$ resulted in no measurable detrimental effects.

\subsection{Neck model}

Figure 1 shows the silicon-based neck model simulator that we developed. A frontal comparison is shown in Figs. 2(a) and 2(b), which are sonographic images of the thyroid using this simulator and in a human subject, respectively. The following observations were made from this comparison.

- The base material can only project to a depth of $2 \mathrm{~cm}$.

- The distinction between the component/organ boundary and the grayscale can be recognized.

- The sodium phosphate used in the thyroid resembles the muscle layer in the human subject image.

- Aside from the muscle layer, applying grayscale changes to calcium phosphate used in the subcutaneous tissue makes it more closely resemble the human subject image.

- In the human subject, the thyroid appears whiter than the subcutaneous tissue.

- The blood vessels, trachea, and spine closely resemble the human subject image.

Next, puncture verification was performed. The echo image at the time of puncture is shown in Fig. 3. The following observations were made.

- A very small trace of the needles remains.

- In regard to haptic realism, all organs and components closely simulate human subjects during puncture with the exception of the blood vessels, which are harder than those of human subjects.

\section{Validation}

To evaluate how much the simulator that we developed was different from the actual human body structure, we conducted a questionnaire to survey 10 doctors with different years of experience. The contents of the questionnaire are shown below.

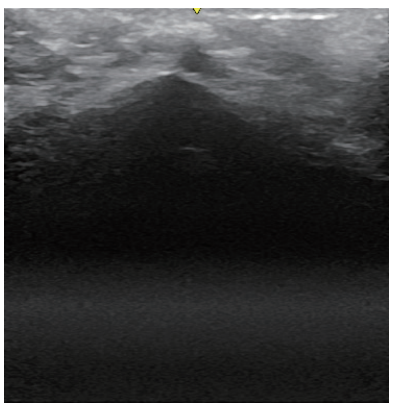

(a)

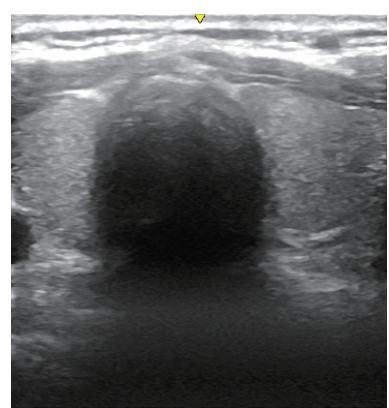

(b)

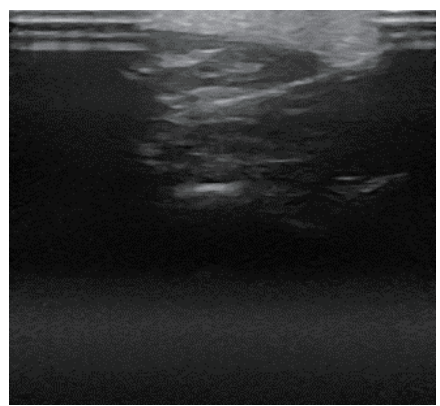

Fig. 2. Sonographic images of the thyroid (a) using this simulator and (b) in a human subject.

Fig. 3. The echo image at the time of puncture. 
Question 1: Feel when lightly pressing the probe

Question 2: Rapid recognition of the thyroid

Question 3: Elasticity of the organization when the probe is strongly pressed

Question 4: Feel when the needle is stabbed

Question 5: How echoes look when the needle passes through the simulator

Evaluation is made in the following three stages.

0 : Deviation from the actual product is large and inappropriate for training.

1: Although there is a deviation from the real thing, it can be used for training.

2: Close to the real thing, suitable for training.

Table 3 shows the questionnaire results. From this questionnaire, we found the following:

- There is no difference according to the years of doctor experience.

- The recognition rate of anatomical structures is good.

- Hardness is higher than actual and there is a difference in feel.

- It is difficult to recognize the needle because the echo transmission distance is limited to $2 \mathrm{~cm}$.

\section{Discussion}

A medical imaging diagnostic apparatus provides the visualization of the inside of the human body that cannot be directly viewed and presents it to the doctor. This is a very important tool that is combined with the doctor's experience and intuition, making the diagnosis of medical abnormalities possible. An ultrasonic diagnostic apparatus is frequently used in medical fields because it is easy and convenient to use. There are two important points in the acquisition of and diagnosis using ultrasound diagnostic device data. One is to understand the tissue structure in the body and the tomograms observed by various operations of the ultrasonic probe, and the other is to obtain the tomograms necessary to diagnose any given medical disorder. To master these techniques, in addition to acquiring knowledge of the characteristics of ultrasound and the structure of the human body, it is necessary to actually operate the ultrasound diagnostic apparatus and observe a large number of tomograms. Although it is

Table 3

Questionnaire results.

\begin{tabular}{lcllllll}
\hline & $\begin{array}{c}\text { Doctor experience } \\
\text { years }\end{array}$ & Q.1 & Q.2 & Q.3 & Q.4 & Q.5 & Total \\
\hline Respondent 1 & 3 & 1 & 2 & 2 & 1 & 0 & 6 \\
Respondent 2 & 9 & 1 & 2 & 1 & 0 & 1 & 5 \\
Respondent 3 & 9 & 1 & 2 & 1 & 2 & 1 & 7 \\
Respondent 4 & 9 & 2 & 1 & 2 & 1 & 0 & 6 \\
Respondent 5 & 10 & 1 & 1 & 1 & 1 & 2 & 6 \\
Respondent 6 & 10 & 1 & 2 & 1 & 1 & 1 & 6 \\
Respondent 7 & 11 & 1 & 2 & 1 & 1 & 1 & 6 \\
Respondent 8 & 11 & 1 & 2 & 1 & 1 & 0 & 5 \\
Respondent 9 & 13 & 2 & 2 & 2 & 2 & 1 & 9 \\
Respondent 10 & 13 & 1 & 1 & 1 & 1 & 0 & 4 \\
\hline Average & 9.8 & 1.2 & 1.7 & 1.3 & 1.1 & 0.7 & 6 \\
\hline
\end{tabular}


desirable to practice on the target patients to improve ultrasound diagnostic skills, there is a limit to patient availability and cooperation, and there is a need for a training method that does not require patients. Therefore, various phantoms have been created, but the tomograms presented to trainees to date cannot accurately reproduce the internal anatomy. To reproduce this accurately, materials with appropriate characteristics (acoustic impedance) are injected into 3D-printed molds to create each component/organ together creating a sophisticated phantom. This makes it possible to visualize direction-dependent ultrasonic tomograms similar to those expected from actual patients. In previous studies, phantoms using tofu, ${ }^{(2)}$ gelatin, ${ }^{(3)}$ agar, ${ }^{(1,4)}$ silicone, ${ }^{(5)}$ and cadavers ${ }^{(6)}$ have been reported; however, in this study, silicon-based materials were used in consideration of cost and long-term availability.

In this experiment, the frequencies used ranged from 7.5 to $10 \mathrm{MHz}$. Ultrasonic wave bursts of about 1 to $30 \mathrm{~Hz}$ are often used for medical diagnosis. In ultrasonic diagnosis, the spatial resolution improves with higher frequencies. However, the ability to penetrate below the surface is inversely related as higher frequencies attenuate rapidly below the surface. Therefore, it is important to select an appropriate frequency for any given target area/depth. Although the thyroid gland is a superficial organ, the frequency needs to be low because the recognition of the positional relationship with the deeper trachea, blood vessels, and vertebral bodies is also essential in actual medical practice.

The acoustic impedance indicates the hardness or resistance to the passage of ultrasonic waves and is calculated as

$Z($ acoustic impedance $)=P($ density of substance $) \times C$ (sound velocity specific to substance $)$

The acoustic impedance of each living tissue is shown in Table 4. To create materials with different acoustic impedances, the density of silicon is stratified by mixing compounds to create materials with acoustic impedances similar to that of biological tissue. Moreover, it was also possible to render variations in internal texture by varying the particle size of the compound used. The ultrasound transmitted from the probe into the body is reflected depending on the difference in acoustic impedance between the components/organs, and the greater the difference in acoustic impedance, the greater the reflection, whereas little variation in acoustic impedance between components/organs results in less reflection. Compared with organs, the acoustic impedance of air is extremely low and that of bones is extremely high, so organs and bones containing air, such as lungs and intestines, are likely to be highly reflective and be

Table 4

Acoustic impedance of each living tissue.

\begin{tabular}{lccc}
\hline & Speed of Sound $(\mathrm{m} / \mathrm{s})$ & Density $\left(\mathrm{g} / \mathrm{cm}^{2}\right)$ & Acoustic impedance $\left(10^{6} \mathrm{~kg} / \mathrm{m}^{2} \cdot \mathrm{s}\right)$ \\
\hline Air & 344 & 0.0012 & 0.0004 \\
Fat & 1476 & 0.92 & 1.36 \\
Water & 1500 & 0.997 & 1.495 \\
Blood & 1560 & 1.06 & 1.65 \\
Muscle & 1568 & 1.06 & 1.65 \\
Bone & 3300 & 1.6 & 5.28 \\
\hline
\end{tabular}


resistant to the permeation of sound. It is also possible to create an artifact artificially by the selection of materials. Artifacts in ultrasound often provide important clues to the detection of abnormalities. On the other hand, if attention is not paid to the direction of the ultrasound, it may become a factor that hinders an accurate understanding of the biological structure. This is an important step in learning ultrasound imaging and is essential in the medical field. The creation of phantoms customized to specific cases was clearly discussed in previous work. Such a phantom could be used as a base model, and the addition of a variety of artifacts could simulate a wide variety of case studies.

\section{Conclusions}

This phantom is a prototype and continued development is under way. In this study, the above experiments confirmed the effectiveness of the silicon-based model and the use of additional compounds. Although some oil bleed occurs on the current silicon-based model, specialized storage is no longer required. In addition, the haptic feedback during the puncture procedure is realistic and the needle leaves a very small trace after use. A significant limitation of the current model is that the echo-transmittable distance is limited to $2 \mathrm{~cm}$. This needs to be extended to 5 to $6 \mathrm{~cm}$ to be fully representative of a human patient. To increase this depth, a different type of silicone is required.

\section{Acknowledgments}

This development was made possible by the collaboration between Nagasaki University and MICOTO Technology Co., Ltd.

\section{References}

1 M. Baba, K. Matsumoto, N. Yamasaki, H. Shindo, H. Yano, M. Matsumoto, R. Otsubo, M. J. Lawn, N. Matsuo, I. Yamamoto, S. Hidaka, and T. Nagayasu: J. Surg. Edu. 74 (2017) 1039.

2 K. Nattagh, T. Siauw, J. Pouliot, I. C. Hsu, and J. A. Cunha: Brachytherapy 13 (2014) 413.

3 A. A. Gresens, R. C. Britt, E. C. Feliberti, and L. D. Britt: J. Surg. Edu. 69 (2012) 411.

4 K. Sugimoto, F. Moriyasu, J. Shiraishi, M. Yamada, and Y. Imai: AJR 196 (2011) 429.

5 M. C. Frates, C. B. Benson, J. W. Charboneau, E. S. Cibas, O. H. Clark, B. G. Coleman, J. J. Cronan, P. M. Doubilet, D. B. Evans, J. R. Goellner, I. D. Hay, B. S. Hertzberg, C. M. Intenzo, R. B. Jeffrey, J. E. Langer, P. R. Larsen, S. J. Mandel, W. D. Middleton, C. C. Reading, S. I. Sherman, and F. N. Tessler: Radiology 237 (2005) 794.

6 E. S. Cibas, E. K. Alexander, and C. B. Benson: Diagn. Cytopathol. 36 (2008) 390. 